\title{
Análisis de los índices económicos en Ecuador y su afectación por la crisis sanitaria en los dos primeros trimestres del año 2020
}

\author{
Ximena Yadira Naranjo Ruiz \\ ximena.naranjo@unl.edu.ec \\ Universidad Nacional de Loja \\ Andrés Roberto Navas Castellanos \\ andres.navas@unl.edu.ec \\ Universidad Nacional de Loja \\ Talia Elizabeth Riascos Eras \\ talia.riascos@unl.edu.ec \\ Universidad Nacional de Loja \\ Franklin Yovani Malla Alvarado \\ franklin.malla@unl.edu.ec \\ Universidad Nacional de Loja
}

\section{RESUMEN}

La presente investigación permite apreciar el impacto económico que deja la crisis sanitaria en el país y que se ve reflejada en varios indicadores macroeconómicos como el producto interno bruto PIB, empleo, exportaciones entre otros, que han dejado secuelas o dificultades de solventar en el corto plazo y para lo cual la administración del gobierno deberá plantear las estrategias permitentes para aliviar las contingencias negativas de la crisis que afecta a nivel mundial y sin una solución definitiva; entre los principales resultados se puede mencionar que la capacidad de crear bienes y servicios PIB se ve disminuido gradualmente en los dos primeros trimestres del año 2020, afectando de manera directa en la entrada de divisas al estado por concepto de tributos y la disminución de plazas de empleo tan necesarios en tiempos de aislamiento.

Palabras clave: impacto económico; indicadores; bienes y servicios; empleo. 


\title{
Analysis of economic indices in Ecuador and their affection by the health crisis in the first two quarters of the year 2020
}

\begin{abstract}
This research allows us to appreciate the economic impact left by the health crisis in the country and which is reflected in several macroeconomic indicators such as gross domestic product, GDP, employment, exports, among others, which have caused difficulties in solving in the short term and for which the government administration must propose the permitting strategies to alleviate the negative contingencies of the crisis that affects worldwide and without a definitive solution; Among the main results, it can be mentioned that the ability to create goods and services GDP is gradually diminished in the first two quarters of 2020, directly affecting the entry of foreign currency to the state by way of taxes and the decrease in jobs much needed employment in times of isolation.
\end{abstract}

Keywords: economic impact; indicators; goods and services; employment.

Artículo recibido: 20 diciembre. 2021 Aceptado para publicación: 10 enero 2022 Correspondencia: ximena.naranjo@unl.edu.ec Conflictos de Interés: Ninguna que declarar 


\section{INTRODUCCIÓN}

Para que una administración del estado pueda desempeñar sus funciones y programas sociales de manera óptima, requiere de los recursos necesarios para financiarlos, ahí la importancia de contar con flujos de efectivo que permitan solventarlos, brindando servicios de calidad a toda la población; en este sentido Chafla, Suárez y Gil (2020) manifiestan, en alusión a lo que señalan las directrices del Ministerio de Economía y Finanzas, que estos ingresos pueden ser de dos categorías, permanentes y no permanentes; los permanentes son aquellos recursos públicos que el estado adquiere a través de sus entidades o instituciones a nivel nacional, los ingresos no permanentes en cambio, son entradas de efectivo que el gobierno percibe por situaciones específicas o extraordinarias.

Por su parte García y Tobar (2019) señalan que los ingresos del estado son petroleros y no petroleros, sumados al resultado operacional de las empresas públicas. Estos datos son muy importantes ya que la investigación gira en torno a cómo ha sido la economía del Ecuador en los dos primeros trimestres del año 2020 en relación a los años anteriores, y qué medidas se pueden establecer para minimizar el impacto en todos los sectores socioeconómicos; recordando también que la crisis sanitaria afectó a todo el mundo y por lo tanto a aliados estratégicos de la economía ecuatoriana en cuanto a las exportaciones de productos tradicionales y no tradicionales.

También resulta imperante mencionar que el sector empresarial ha experimentado una tendencia a la baja muy importante en sus ventas, lo que repercute directamente en la entrada de divisas al estado por concepto de impuestos y la pérdida de puestos de trabajo de miles de personas que tuvieron que ser separadas debido a que las unidades económicas no contaban con el presupuesto necesario para cubrir sus costes operativos. Para confrontar los resultados que son presentados en su parte pertinente, se analizan los siguientes conceptos:

\section{Producto interno bruto PIB}

El producto interno bruto que fue inventado en la década de 1940, es un indicador clave de la política económica de los países, es un referente para la medición de la magnitud de la economía de una nación, y es habitual que lo relacione con otros indicadores de crecimiento muy importantes como el déficit fiscal y el índice de desarrollo humano (Uhsca, Andrade y Riquero, 2019). Uno de los usos más frecuentes del PIB es como 
medida de bienestar material, su cálculo es complejo y siempre está presente en las contabilidades de los países.

Es una magnitud macroeconómica que permite medir el valor monetario de todos los bienes y servicios comprados por el consumidor final, los mismos que son elaborados o producidos en un país en un tiempo determinado, pudiéndose decir que el PIB representa el tamaño de la economía de un país (Borsic, Benítez y García 2016). Este indicador mide el ritmo en el que aumenta la producción de bienes o servicios, teniendo la facilidad de desglosarse en términos de la contribución de cada sector económico de una nación en particular. Cuando este indicador macroeconómico presenta tendencias de crecimiento, es una señal clara que dentro del país donde se ejecuta, se están brindando mayores oportunidades para que todos los sectores que se relaciona con la economía puedan desarrollar sus actividades en un marco legal establecido y óptimo.

\section{Exportaciones}

A través de la globalización del contexto empresarial, se ha logrado la apertura de oportunidades muy importantes para el desarrollo económico de las organizaciones a través de su internacionalización; dicho esto, las exportaciones son una forma que tienen las empresas para participar en mercados internacionales, requiriendo una inversión considerable pero flexibilizando sus actividades y posibilitando en un porcentaje mayor la maximización de sus inversiones (Escandón, Ayala y Caicedo, 2018).

Las exportaciones pueden ser desagregadas como de bienes primarios sin petróleo y petroleras; tradicionales y no tradicionales, esto dependiendo del tratamiento que se les dé en un país o región (Reyes y Jumenez, 2012); sin olvidar que los países que son más abiertos al comercio internacional, son precisamente los que tienden a tener un mayor crecimiento económico, de ahí la importancia de eliminar barreras al comercio y la creación de políticas que fomenten su ejecución (Cáceres, 2013).

\section{Inversión extranjera}

La inversión extranjera directa promueve el crecimiento de un país a través del incremento de la acumulación de capital en el país receptor por medio de insumos y nuevas tecnologías o transfiere tecnología de empresas extranjeras a las nacionales, desempeñando un papel importante en el crecimiento de las economías en desarrollo (Suanes y Roca, 2015). 
En su estudio Rivas y Puebla (2016) manifiestan que la inversión extranjera es muy sensible a los ciclos económicos de las naciones (épocas buenas y también los no tan buenos o crisis económicas) y esta, precisamente puede ser la razón porque las empresas prefieren ser una forma internacional a una doméstica, ya que tendrán mayores oportunidades de solventar situaciones o contingencias adversas.

\section{Impuestos}

"La tributación es la parte central de la política fiscal" (Barros, 2013, p. 38); de ahí que los impuestos, al ser parte de los tributos, son una herramienta eficaz para conseguir recursos por la actividad económica que desarrollan los contribuyentes y poder canalizarlo en el gasto social. Son uno de los instrumentos de mayor importancia con los que cuenta el estado para fortalecer el desarrollo económico, ya que a través de ellos se puede influenciar en los niveles de asignación del ingreso entre toda la población, dependiendo en gran medida del nivel de recaudación alcanzado (Cayo, 2010).

Es importante resaltar que cuando una administración del estado decide ser más flexible con los impuestos, su propósito se resume en mejorar la competitividad del país, incrementar de forma significativa los flujos de la inversión directa, convertirse en un régimen impositivo más simple y eficiente, eliminando gran parte de las distorsiones en la tributación sectorial (Ávila y León, 2010). Esta reflexión es significativa desde el punto de vista actual de las empresas, que requieren de minorar su carga tributaria para lograr una recuperación económica pertinente con la realidad y futuro inmediato que les toca afrontar. Los impuestos pueden ser directos como el impuesto a la renta o indirectos como el impuesto al valor agregado, que afecta a todos los procesos de comercialización de un producto.

\section{Empleo}

El trabajo es un factor elemental en la vida del ser humano, representa la relación entre los seres humanos y la naturaleza, de donde se obtienen los bienes y dan la forma a las interacciones sociales, proporcionado a su vez, las bases de la producción económica que se refleja en toda la visa social y política (Sousa, 2012).

Un elemento clave en la empleabilidad es la calidad de vida laboral, ante ello, los factores que influyen para que esta calidad sea la adecuada, son los siguientes: 
- Condiciones ambientales: que involucra los aspectos relacionados con la satisfacción laboral, los riesgos laborales y la carga de trabajo.

- Condiciones ergonómicas: relacionadas con el sitio de trabajo, es decir el bienestar que les ofrece.

- Condiciones económicas: que se relacionan directamente a la proporción de dinero que recibe el trabajador por el desarrollo de sus actividades dentro de la empresa y que le permita cubrir sus necesidades (Martínez, Oviedo y Luna, 2013).

\section{Crisis sanitaria}

La pandemia del COVID 19 (SARS- CoV-2) tomó a la humanidad por sorpresa, al tratarse de un organismo nuevo, con un elevadísimo porcentaje de propagación y sobre todo por su manejo complejo en sus diferentes fases de presentación, sumado a ello el desabasto de recurso médicos en todas las naciones, su falta de tratamiento y las múltiples repercusiones psicológicas, económicas y políticas (Casas, 2020).

\section{ESTRATEGIAS METODOLÓGICAS O MATERIALES Y MÉTODOS}

La investigación es de tipo documental, utilizando la revisión de las páginas oficiales del Banco Central del Ecuador, Servicio de Rentas Internas, Instituto Ecuatoriano de Estadísticas y Censos y otros, permitieron verificar la afectación de la crisis sanitaria en el contexto económico del país, posibilitando el planteamiento de posibles alternativas para solventar en parte la crisis actual.

El método usado es la descripción con el fin de detallar de forma clara cuál ha sido el comportamiento de los indicadores analizados y como afectan la economía local; de la misma forma el análisis resultó imprescindible para comprender cómo la crisis sanitaria no solo es médica, sino que hizo su impacto en todos los sectores sociales, políticos y económicos del mundo entero, siendo hasta el día la preocupación de todas las naciones y organismos multilaterales.

\section{RESULTADOS Y DISCUSIÓN}

Esta parte incluye los cuadros y gráficos de los principales indicadores macroeconómicos del país en relación a los mismos periodos de años anteriores, evidenciando el impacto de la crisis sanitaria. 
Tabla 1: PIB trimestral

\begin{tabular}{|c|c|c|c|}
\hline Año & Trimestre & $\begin{array}{l}\text { PIB TRIMESTRAL Miles de } \\
\text { dólares (Constantes) }\end{array}$ & $\begin{array}{c}\text { TASAS DE } \\
\text { VARIACIÓN t/t-4 } \\
\text { (Constantes) }\end{array}$ \\
\hline \multirow{4}{*}{2017} & $\mathrm{~T} 1$ & 17.497 .935 & 1,7 \\
\hline & $\mathrm{T} 2$ & 17.685 .968 & 2,1 \\
\hline & T3 & 17.819 .405 & 2,9 \\
\hline & $\mathrm{T} 4$ & 17.952 .383 & 2,8 \\
\hline \multirow{4}{*}{2018} & $\mathrm{~T} 1$ & 17.762 .564 & 1,5 \\
\hline & $\mathrm{T} 2$ & 17.943 .194 & 1,5 \\
\hline & T3 & 18.080 .826 & 1,5 \\
\hline & $\mathrm{T} 4$ & 18.083 .933 & 0,7 \\
\hline \multirow{4}{*}{2019} & $\mathrm{~T} 1$ & 17.957 .733 & 1,1 \\
\hline & $\mathrm{T} 2$ & 18.028 .980 & 0,5 \\
\hline & T3 & 18.022 .042 & $-0,3$ \\
\hline & $\mathrm{T} 4$ & 17.900 .370 & -1 \\
\hline \multirow{2}{*}{2020} & $\mathrm{~T} 1$ & 17.537 .974 & $-2,3$ \\
\hline & $\mathrm{T} 2$ & 15.789 .815 & $-12,4$ \\
\hline \multicolumn{4}{|c|}{ Fuente: Banco Central del Ecuador } \\
\hline
\end{tabular}

Figura 1: PIB trimestral $2017-2020$

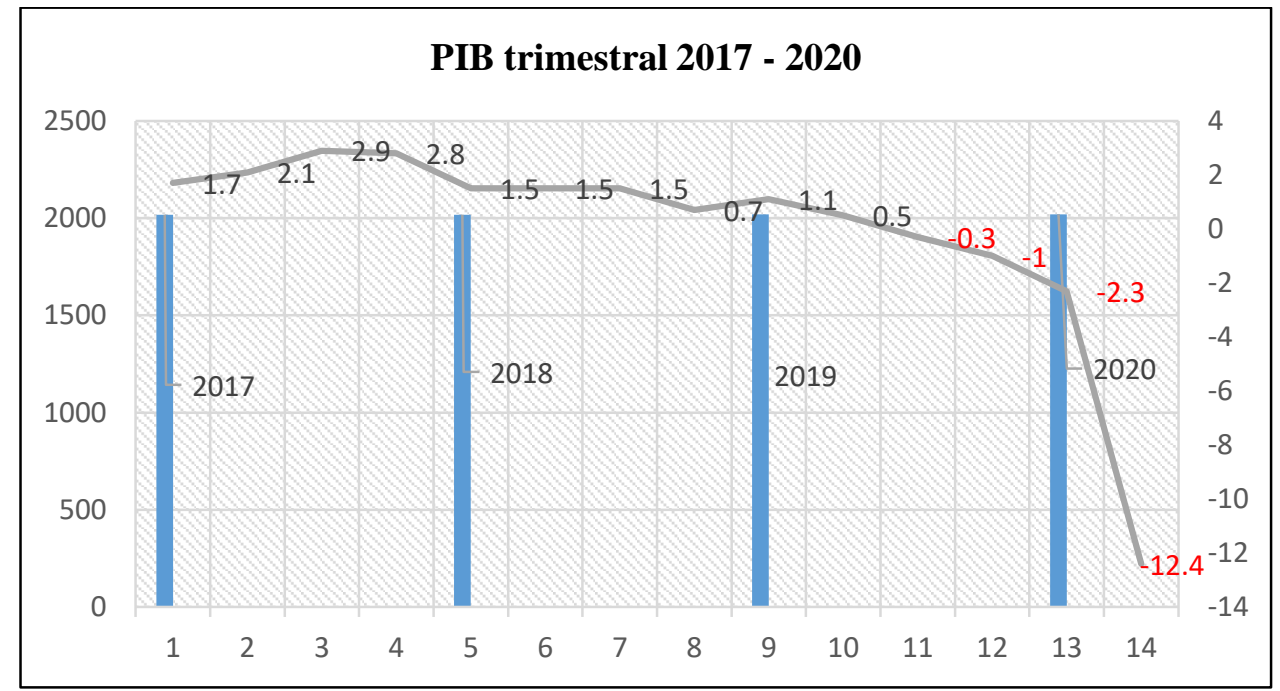

Fuente: Banco Central del Ecuador

\section{Análisis}

Como se puede apreciar el impacto de la crisis sanitaria golpea duramente en los dos primeros trimestres del 2020, donde se reflejan disminuciones importantes de la capacidad de producir bienes y servicios para el consumo de la población; cabe recalcar que la tendencia a la baja del PIB ya empieza años anteriores `pero se agudiza en el año 
actual debido al cierre de empresas que fabrican los productos por la poca demanda que existió y existe en el mercado nacional, obligándolos a estancar sus niveles de producción a la espera de encontrar una solución viable a la problemática de la salud.

Tabla 2: Exportaciones y destinos

\begin{tabular}{|c|c|c|c|c|c|c|c|}
\hline Año & Indicador & África & América & Asia & Europa & Oceanía & $\begin{array}{c}\% \\
\text { América }\end{array}$ \\
\hline \multirow{4}{*}{$\begin{array}{l}\text { AÑO } \\
2017\end{array}$} & FOB T 1 & $8.664,50$ & $2.785 .604,20$ & $819.498,50$ & $1.069 .501,40$ & $16.725,50$ & \\
\hline & FOB T 2 & $10.608,30$ & $2.707 .267,30$ & $866.008,30$ & $1.054 .213,30$ & $14.444,50$ & $-2,81 \%$ \\
\hline & FOB T 3 & $8.463,60$ & $2.703 .970,90$ & $904.145,10$ & $1.001 .648,50$ & $12.842,40$ & $-0,12 \%$ \\
\hline & FOB T 4 & $15.715,00$ & 3.035 .0 & $\overline{001.4}$ & 1.03 & $5.105,00$ & $12,24 \%$ \\
\hline \multirow{4}{*}{$\begin{array}{l}\text { AÑO } \\
2018\end{array}$} & דו & $22.0+5,00$ & $2.971 .452,10$ & $1.095 .296,70$ & $1.095 .635,80$ & $4.578,40$ & $-2,09 \%$ \\
\hline & FOB T 2 & $20.531,00$ & $3.313 .134,20$ & $1.067 .118,50$ & $1.111 .217,40$ & $12.745,90$ & $11,50 \%$ \\
\hline & FOB T 3 & $16.721,50$ & $3.450 .313,90$ & $1.044 .898,60$ & $1.031 .847,90$ & $14.720,30$ & $4,14 \%$ \\
\hline & FOB T 4 & $22.456,60$ & $3.175 .789,70$ & 1.076. & 1.048 & ,20 & $-7,96 \%$ \\
\hline \multirow{4}{*}{$\begin{array}{l}\text { AÑO } \\
2019\end{array}$} & FOB T 1 & $28.142,00$ & $3.188 .607,50$ & $982.761,90$ & $1.029 .461,70$ & $14.548,90$ & $0,40 \%$ \\
\hline & FOB T 2 & $45.720,30$ & $3.367 .078,80$ & $1.314 .699,90$ & $1.021 .945,90$ & $12.807,90$ & $5,60 \%$ \\
\hline & FOB T 3 & $33.312,60$ & $3.446 .689,50$ & $1.143 .478,20$ & $1.044 .184,50$ & $12.967,40$ & $2,36 \%$ \\
\hline & FOB T 4 & $45.504,80$ & $3.142 .654,50$ & $1.392 .654,10$ & $1.047 .202,80$ & $11.454,00$ & $-8,82 \%$ \\
\hline \multirow{3}{*}{$\begin{array}{l}\text { AÑO } \\
2020\end{array}$} & FOB T 1 & $33.435,20$ & $2.755 .292,80$ & $1.206 .058,00$ & $1.309 .741,20$ & $12.187,30$ & $-12,33 \%$ \\
\hline & FOB T 2 & $64.913,60$ & $1.784 .114,40$ & $1.371 .747,60$ & $953.878,70$ & $14.820,80$ & $-35,25 \%$ \\
\hline & FOB T 3 & $58.102,90$ & $2.810 .555,20$ & $1.072 .088,10$ & $1.190 .772,60$ & $16.353,00$ & $57,53 \%$ \\
\hline
\end{tabular}

Figura 2: Tendencias de las exportaciones: América

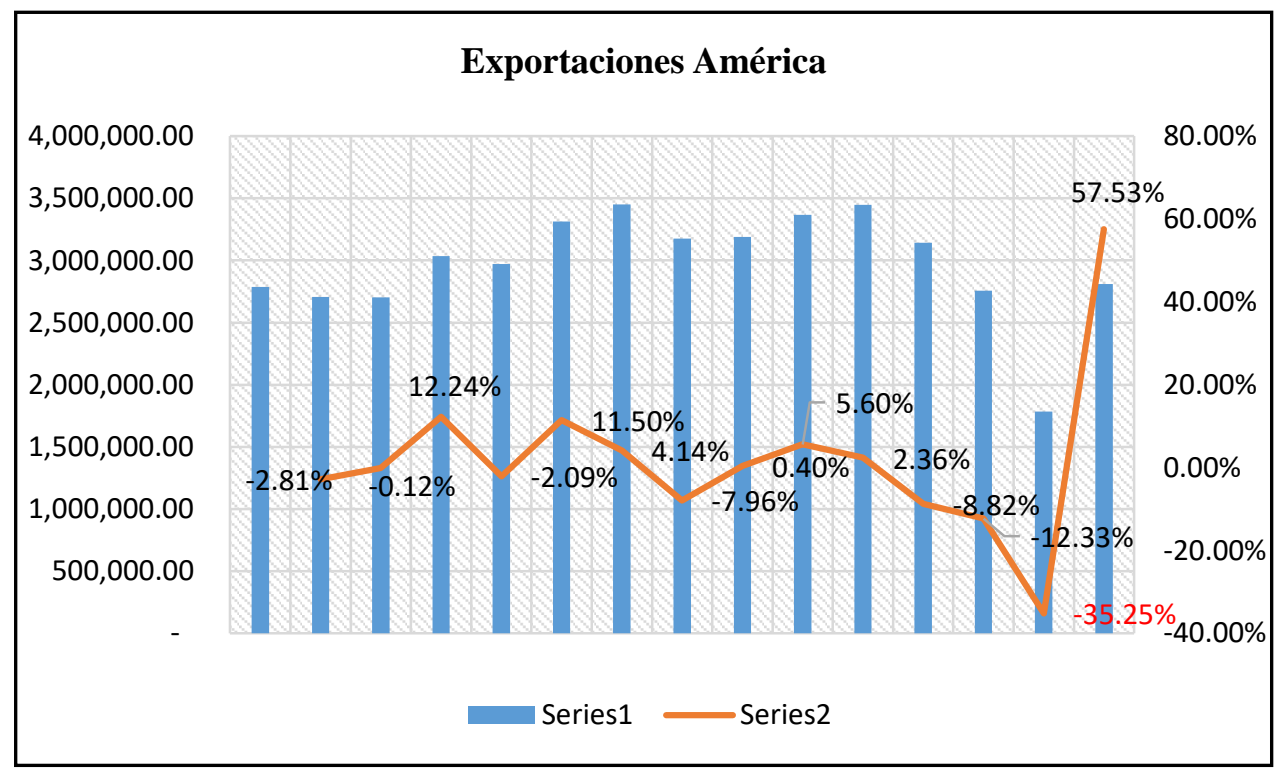

Fuente: Banco Central del Ecuador 


\section{Análisis}

El sector exportador fue uno de los pocos que continuó con sus operaciones habituales (pero si en menor cantidad) debido a los compromisos que se tiene con las empresas internacionales, de ahí que las autoridades locales permitieron la salida de vuelos internacionales por estos rubros y no debilitar en parte la economía interna; cabe recalcar que el segundo trimestre del 2020 fue el más golpeado con una disminución del 35,25\% (con destino a América por ser el más fuerte para el país) en virtud que fueron los meses donde más se complicó la crisis sanitaria y por lo tanto los controles se incrementaron en algunos destinos de las exportaciones. Para el tercer trimestre del 2020 se observa una recuperación del 57,53\% hacia el mismo destino, en relación a la apertura de aeropuertos y vías marítimas que permanecían cerradas.

Tabla 3: Inversión directa por país o región

\begin{tabular}{|c|c|c|c|c|c|c|c|c|}
\hline \multicolumn{2}{|c|}{$\begin{array}{c}\text { Año / } \\
\text { Trimestre } \\
\end{array}$} & $\begin{array}{l}\text { América } \\
\text { EE.UU. }\end{array}$ & $\begin{array}{c}\text { Comunidad } \\
\text { Andina } \\
\end{array}$ & $\begin{array}{l}\text { Resto De } \\
\text { América }\end{array}$ & Europa & Asia & Oceanía & $\begin{array}{c}\% \\
\text { América } \\
\end{array}$ \\
\hline \multirow{4}{*}{$\begin{array}{l}\text { AÑO } \\
2017\end{array}$} & $\mathrm{~T} 1$ & $141.547,87$ & $14.054,22$ & $115.617,21$ & $26.035,78$ & $14.746,15$ & $4.238,31$ & \\
\hline & $\mathrm{T} 2$ & $51.022,55$ & $8.106,28$ & $28.170,37$ & $52.069,68$ & $34.218,66$ & $19.921,31$ & $-63,95 \%$ \\
\hline & $\mathrm{T} 3$ & $46.131,85$ & $3.898,12$ & $40.260,92$ & $34.934,27$ & $35.470,27$ & $34.338,94$ & $-9,59 \%$ \\
\hline & $\mathrm{T} 4$ & $6.559,39$ & $5.681,12$ & $-5.581,52$ & $26.325,71$ & $19.993,09$ & $45.445,83$ & $-85,78 \%$ \\
\hline \multirow{4}{*}{$\begin{array}{l}\text { AÑO } \\
2018\end{array}$} & $\mathrm{~T} 1$ & $193.842,22$ & $80.795,67$ & $100.495,67$ & $118.956,91$ & $13.552,74$ & 1,60 & $2855,19 \%$ \\
\hline & $\mathrm{T} 2$ & $132.779,20$ & $-4.659,40$ & $129.750,97$ & $66.448,75$ & $20.877,45$ & 1,88 & $-31,50 \%$ \\
\hline & $\mathrm{T} 3$ & $138.865,71$ & $12.995,35$ & $123.314,74$ & $88.661,52$ & $19.022,25$ & 7,05 & $4,58 \%$ \\
\hline & $\mathrm{T} 4$ & $416.047,75$ & $8.234,82$ & $370.623,76$ & $173.515,11$ & $14.855,14$ & 793,30 & $199,60 \%$ \\
\hline \multirow{4}{*}{$\begin{array}{l}\text { AÑO } \\
2019\end{array}$} & $\mathrm{~T} 1$ & $158.045,70$ & $7.957,04$ & $133.664,06$ & $17.322,41$ & $33.115,79$ & $-521,00$ & $-62,01 \%$ \\
\hline & $\mathrm{T} 2$ & $100.540,00$ & $10.284,63$ & $86.215,14$ & $154.304,62$ & $-3.059,06$ & $23.996,83$ & $-36,39 \%$ \\
\hline & $\mathrm{T} 3$ & $84.785,21$ & $4.220,09$ & $67.062,05$ & $44.165,67$ & $-623,73$ & 250,00 & $-15,67 \%$ \\
\hline & $\mathrm{T} 4$ & $157.358,69$ & $8.409,53$ & $108.540,20$ & $137.125,68$ & $5.514,12$ & $14.616,00$ & $85,60 \%$ \\
\hline \multirow{2}{*}{$\begin{array}{l}\text { ANO } \\
2020\end{array}$} & $\mathrm{~T} 1$ & $123.784,60$ & $10.113,89$ & $52.693,57$ & $97.878,06$ & $6.714,21$ & 0,80 & $-21,34 \%$ \\
\hline & $\mathrm{T} 2$ & $2.631,86$ & $-193,84$ & $-3.586,27$ & $76.298,88$ & $15.740,33$ & - & $-97,87 \%$ \\
\hline \multicolumn{9}{|c|}{ Fuente: Banco Central del Ecuador } \\
\hline
\end{tabular}


Figura 3: Inversión directa EEUU

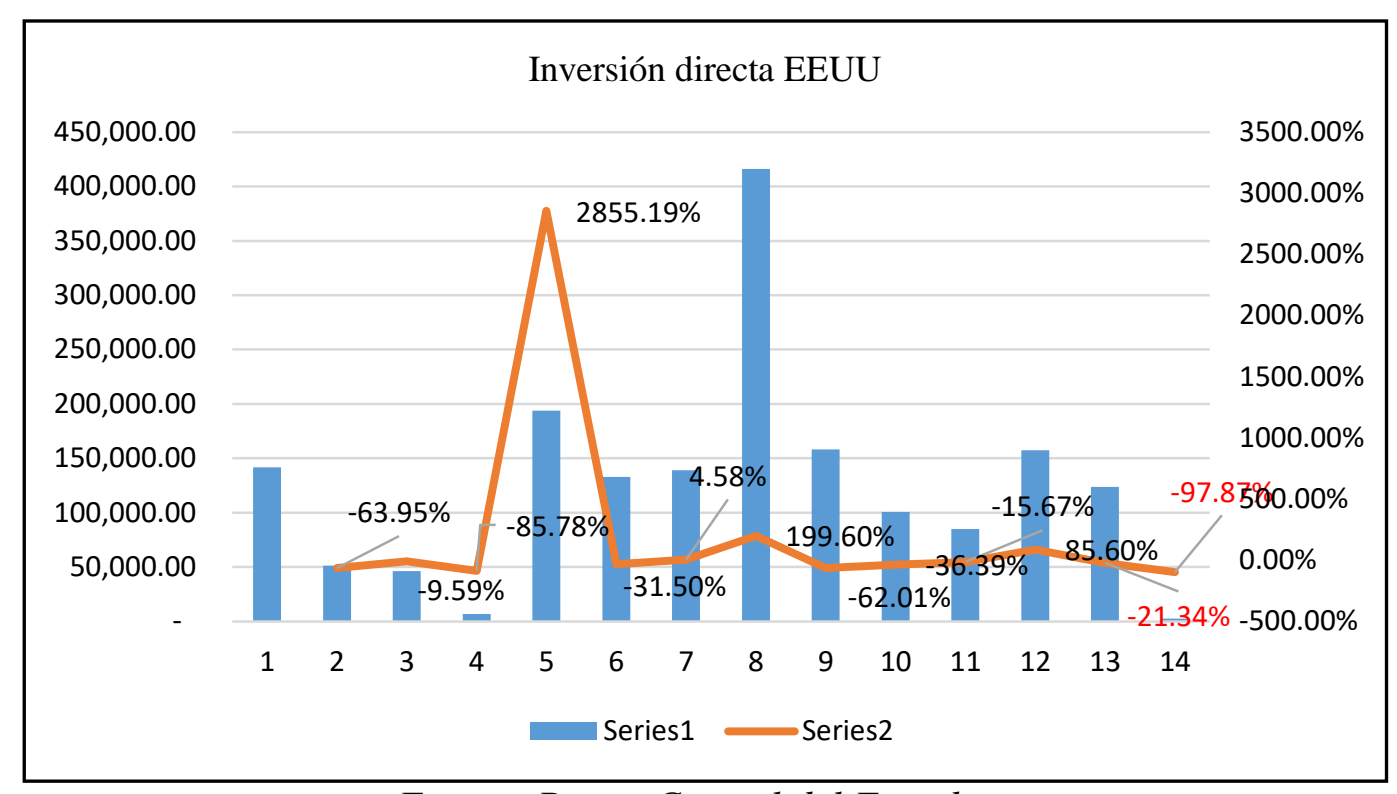

Fuente: Banco Central del Ecuador

\section{Análisis}

La inversión directa que tiene que ver con nuevos capitales, tecnología o suministros de otros países, ha sufrido una disminución considerable en el primer trimestre del año 2020 agudizándose más en el segundo donde se tiene una disminución del 97,87\%, es decir; en ese periodo el país no recibió inversión del exterior siendo un duro golpe a la economía, si se considera que esa inversión trae consigo puestos de trabajo e impuestos para las arcas del estado que requieren del financiamiento para solventar sus programas sociales en el corto plazo, más aún con la presencia de la crisis sanitaria.

Tabla 4: empleabilidad en el Ecuador

\begin{tabular}{|c|c|c|c|c|c|c|}
\hline Detalle & Mar-19 & Jun-19 & Sep-19 & Dic-19 & May/Jun 2020 & Sep-20 \\
\hline $\begin{array}{l}\text { Población en edad de } \\
\text { trabajar }\end{array}$ & $12.272 .857,00$ & $12.316 .194,00$ & $12.359 .400,00$ & $12.402 .565,00$ & $12.490 .574,00$ & $12.531 .127,00$ \\
\hline $\begin{array}{l}\text { Población } \\
\text { económicamente activa }\end{array}$ & $8.162 .787,00$ & $8.231 .949,00$ & $8.379 .355,00$ & $8.099 .030,00$ & $7.605 .189,00$ & $7.874 .226,00$ \\
\hline Población con empleo & $7.786 .532,00$ & $7.865 .786,00$ & $7.972 .485,00$ & $7.787 .896,00$ & $6.595 .606,00$ & $7.351 .606,00$ \\
\hline $\begin{array}{l}\text { Empleo adecuado } \\
\text { pleno }\end{array}$ & $3.094 .795,00$ & $3.123 .743,00$ & $3.228 .032,00$ & $3.146 .297,00$ & $1.271 .333,00$ & $2.526 .169,00$ \\
\hline Subempleo & $1.524 .118,00$ & $1.667 .278,00$ & $1.649 .346,00$ & $1.440 .983,00$ & $2.620 .966,00$ & $1.840 .425,00$ \\
\hline Empleo no remunerado & $895.242,00$ & $855.741,00$ & $870.833,00$ & $884.617,00$ & $588.607,00$ & $847.702,00$ \\
\hline Otro empleo no pleno & $2.222 .111,00$ & $2.169 .741,00$ & $2.171 .045,00$ & $2.268 .077,00$ & $1.918 .944,00$ & $2.070 .291,00$ \\
\hline Empleo no clasificado & $50.267,00$ & $49.282,00$ & $53.228,00$ & $47.923,00$ & $195.755,00$ & $67.018,00$ \\
\hline Desempleos & $376.255,00$ & $366.163,00$ & $406.871,00$ & $311.134,00$ & $1.009 .583,00$ & $522.620,00$ \\
\hline $\begin{array}{l}\text { Población } \\
\text { económicamente } \\
\text { inactiva }\end{array}$ & $4.110 .070,00$ & $4.084 .245,00$ & $3.980 .045,00$ & $4.303 .535,00$ & $4.885 .385,00$ & $4.656 .901,00$ \\
\hline
\end{tabular}


Figura 4: Empleos / desempleos

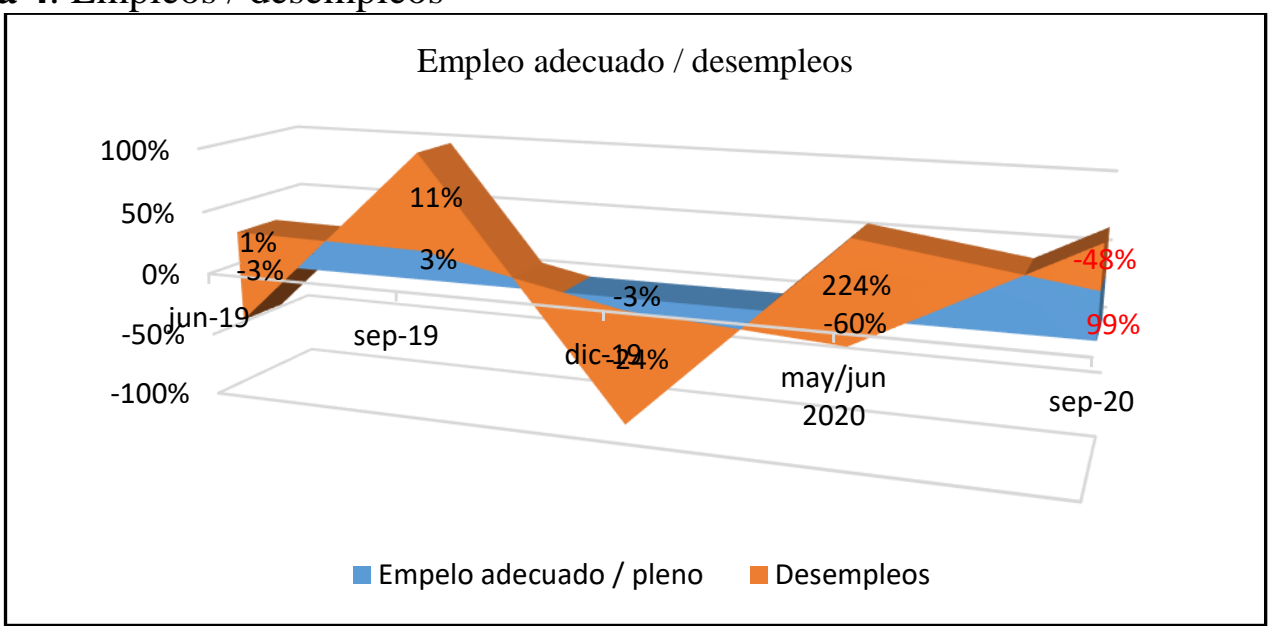

\section{Análisis}

Fuente: Instituto Ecuatoriano de Estadísticas y Censos INEC

Es importante destacar que el empleo adecuado es donde las personas pueden obtener ingresos que les permiten solventar sus necesidades básicas y además contar con un tipo de ahorro; este parámetro presenta una disminución del 60\% de personas con este tipo de empleos para el segundo trimestre del año 2020, evidenciándose una recuperación para septiembre del mismo año que alcanza un crecimiento del $99 \%$ con respectivo al trimestre anterior, es decir junio del 2020. El desempleo por su parte a junio del 2020 señala que hubo un incremento del $224 \%$ debido a que las empresas tuvieron que sacar de sus roles a muchos trabajadores ya que su producción no dejaba niveles de rentabilidad que permita cubrir sus costes; este parámetro también tiene un incremento favorable al tercer trimestre del $48 \%$ lo que evidencia que se abrieron nuevas plazas de empleo con el levantamiento del estado de excepción decretado por el gobierno.

Tabla 5: recaudación de impuestos

\begin{tabular}{|c|c|c|c|}
\hline Año & Trimestre & Impuestos miles de millones & Variación \\
\hline \multirow{4}{*}{2017} & $\mathrm{~T} 1$ & 3,38 & \\
\cline { 2 - 4 } & $\mathrm{T} 2$ & 3,98 & $17,93 \%$ \\
\cline { 2 - 4 } & $\mathrm{T} 3$ & 3,40 & $-14,55 \%$ \\
\cline { 2 - 4 } & $\mathrm{T} 4$ & 3,32 & $-2,43 \%$ \\
\hline \multirow{4}{*}{2018} & $\mathrm{~T} 1$ & 3,28 & $-1,18 \%$ \\
\cline { 2 - 4 } & $\mathrm{T} 2$ & 3,85 & $17,21 \%$ \\
\cline { 2 - 4 } & $\mathrm{T} 3$ & 3,74 & $-2,75 \%$ \\
\cline { 2 - 4 } & $\mathrm{T} 4$ & 2,56 & $-31,53 \%$ \\
\hline \multirow{4}{*}{2019} & $\mathrm{~T} 1$ & 3,35 & $30,64 \%$ \\
\cline { 2 - 4 } & $\mathrm{T} 2$ & 3,73 & $11,32 \%$ \\
\cline { 2 - 4 } & $\mathrm{T} 3$ & 4,00 & $-7,25 \%$ \\
\cline { 2 - 4 } & $\mathrm{T} 4$ & 3,71 & $-7,12 \%$ \\
\hline \multirow{4}{*}{2020} & $\mathrm{~T} 1$ & 3,45 & $-70,28 \%$ \\
\cline { 2 - 4 } & $\mathrm{T} 2$ & 1,02 & \\
\hline
\end{tabular}


Figura 5: Recaudación de impuestos

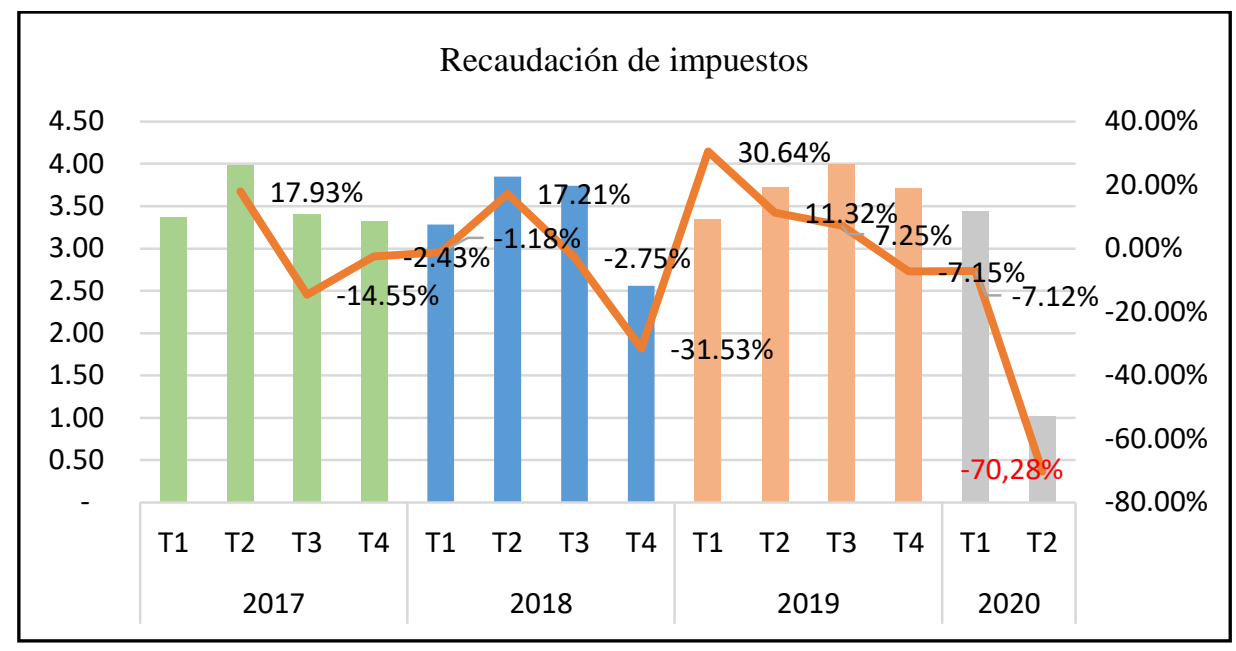

Fuente: Banco Central del Ecuador

\section{Análisis}

Los impuestos que son uno de los aportes más significativos de toda administración del gobierno, en cuanto a la entrada de divisas, también se ve afectado de manera considerable en los primeros trimestres del año 2020, así, en el primer trimestre se ve una reducción del 7,12\% mientras que para el segundo trimestre donde ya se declaró el estado de excepción, las recaudaciones bajaron el 70,28\% de ahí la necesidad del gobierno de buscar alternativas de financiamiento para cubrir su costos sociales.

\section{CONCLUSIÓN O CONSIDERACIONES FINALES}

La crisis sanitaria desatada por el nuevo CORONAVIRUS - 19 tomo de sorpresa a todas las economías del mundo, debiendo adaptarse al ritmo de ir encontrando nuevos tratamientos para solventar la cantidad de afectados en toda la región; en términos económicos su impacto también fue devastador, ya que se declararon estados de excepción en todo el mundo, paralizando empresas en grandes cantidades y por lo tanto creando un estancamiento de la economía globalizada.

En Ecuador y halando de economía, los impactos más duros fueron en los empleos que se perdieron en el segundo trimestre del año 2020, donde muchas personas fueron separadas de sus plazas de trabajo dejándolos sin sustento para sus familias; de la misma forma la inversión extranjera disminuyó su flujo de efectivo y asesoría en tecnología de manera significativa, ya que no se tenían los canales necesarios para su operatividad. Los impuestos por su parte son uno de los indicadores más afectados ya que se observa un menos $70,28 \%$ de recaudación por estos tributos, representado para el estado una grave 
situación en relación al cumplimiento de los programas sociales previamente ya planificados.

Ante esta situación, se puede recomendar a las entidades del gobierno central, ser más flexibles con las medidas económicas que se tomen en la posterioridad, y que las mismas vayan de la mano con la realidad y capacidad de pago de los contribuyentes, logrando con ello un fortalecimiento del sector empresarial y la reactivación de la economía en general que tanto necesita el país.

\section{LISTA DE REFERENCIAS}

Ávila Mahecha, J., \& León Hernández, I. R. (2010). UN IMPUESTO IMPLÍCITO A LA INVERSIÓN. Cuadernos de Economía, 127-146. Obtenido de https://www.redalyc.org/articulo.oa?id=282121980006

Barros Vio, B. (2013). ¿POR QUÉ LAS PERSONAS PAGAN SUS IMPUESTOS? Subjetividad y Procesos Cognitivos, 37-47. Obtenido de https://www.redalyc.org/articulo.oa?id=339630262002

Borsic Laborde, Z., Benítez Burbano, K., \& García Osorio, N. (2016). Análisis de la evolución de la balanza comercial y del producto interno bruto en el Ecuador en el período comprendido entre los años 2011 -2015. Revista de economía y negocios, 56-64. Obtenido de https://revistas.ute.edu.ec/index.php/economia-ynegocios/article/view/175/170

Cáceres Rodríguez, W. (2013). Las exportaciones y el crecimiento económico en Colombia 1994-2010. Revista Apuntes del CENES, 53-80. Obtenido de https://www.redalyc.org/pdf/4795/479549579004.pdf

Casas Martínez, M. d. (2020). Enseñanzas de la pandemia COVID - 19. El reencuentro con la vulnerabilidad humana. Centros Culturales de México, 80-92. Obtenido de https://www.elsevier.es/en-revista-bioethics-update-232-pdf-S2395938X20300206

Cayo Molina, L. J. (2010). EFECTOS ECONÓMICOS DE LA APLICACIÓN DE IMPUESTOS DIRECTOS E INDIRECTOS EN LAS PYMES DEL CANTÓN LATACUNGA; PERÍODO 2008 -2010.”. ESCUELA POLITÉCNICA DEL EJÉRCITO, 1-21. Obtenido de https://repositorio.espe.edu.ec/bitstream/21000/6350/1/AC-ESPEL-CAI-0336.pdf

Chafla Mona, R. A., Suárez López, S. C., \& Gil Espinoza, D. J. (2020). Ingreso vs gasto de gobierno: Evolución del Presupuesto General del Estado, Ecuador, periodo 2013. 
Ciencia \& Tecnologí, 58-78. Obtenido de http://cienciaytecnologia.uteg.edu.ec/revista/index.php/cienciaytecnologia/article/vi ew/275/424

Escandón, D. M., Ayala, A. H., \& Caicedo, M. C. (2018). Influencia de las barreras a la exportación sobre el compromiso exportador y su incidencia en los resultados internacionales. Rev. esc.adm.neg, 38-55. Obtenido de http://www.scielo.org.co/pdf/ean/n75/n75a04.pdf

García Osorio, N. E., \& Tobar Cazares, X. d. (2019). La construcción en el Producto Interno Bruto del Ecuador, 2000 - 2018. PODIUM, 57-69. Obtenido de https://revistas.uees.edu.ec/index.php/Podium/article/view/275/205

Martínez Buelvas, L., Oviedo-Trespalacios, O., \& Luna Amaya, C. (2013). Condiciones de trabajo que impactan en la vida laboral. Salud Uninorte, 542-560. Obtenido de https://www.redalyc.org/pdf/817/81730431015.pdf

Reyes Camargo, S. J., \& Jumenez Ramirez, S. (2012). Composición de las exportaciones y crecimiento económico en la Comunidad Andina de Naciones. Lecturas de Economía, 53-90. Obtenido de http://www.redalyc.org/articulo.oa?id=155226077003

Rivas Aceves, S., \& Donají Puebla Ménez, A. (2016). Inversión Extranjera Directa y Crecimiento Económico. Revista Mexicana de Economía y Finanzas, 51-75. Obtenido de https://www.redalyc.org/pdf/4237/423746532004.pdf

Sousa Santana, V. (2012). Empleo, condiciones de trabajo y salud. Salud Colectiva, 101106. Obtenido de https://www.redalyc.org/pdf/731/73123079007.pdf

Suanes, M., \& Roca-Sagalés, O. (2015). INVERSIÓN EXTRANJERA DIRECTA, CRECIMIENTO ECONÓMICO Y DESIGUALDAD EN AMÉRICA LATINA. $E l$ Trimestre Económico, 675-706. Obtenido de https://www.redalyc.org/pdf/313/31342334006.pdf

Uhsca Cuzco, N., Andrade Valenzuela, P., \& Riquero Castro, H. (2019). Análisis de la correlación entre el PIB y la deuda externa: factior para el crecimiento empresarial. Revista Ciencia eInvestigación, 369-382. Obtenido de file://C:/Users/Usuario/AppData/Local/Temp/DialnetAnalisisDeCorrelacionEntreElPibYLaDeudaExterna-7368591.pdf 